\title{
ПСИХОЛОГИЧЕСКАЯ МОДЕЛЬ ПОВЕДЕНИЯ АГЕНТА
}

\section{PSYCHOLOGICAL MODEL OF BEHAVIOR OF THE AGENT}

\section{S. Kiselev \\ S. Sazonov \\ E. Sazonova}

Summary. Evacuation models used in fire safety technology have significantly expanded their capabilities over the past decades. They have become simple computational tools in which we deal with methods such as overcoming conflicts, while using modern modeling techniques it is possible to model complex behavior and decision making of people. Despite the progress, the methods of evacuation modeling seem obvious, their comparative capabilities for all years, everything that exists, in which there is room for improvement.

Evacuation models used in fire safety techniques have greatly expanded their capabilities over the past decades. They have become simple computational tools in which the equations of motion of people are implemented in discrete spaces, while using modern modeling methods it is possible to simulate complex behavior and decision making by people. Despite the progress of evacuation modeling methods when comparing their capabilities, it seems obvious that there are still many areas in which there is room for improvement.

In evacuation models, the movement of people is usually based on modeling the speed of unhindered walking, with which evacuees move, and the influences on it. This is one of the key factors influencing the results obtained using evacuation models. Currently, various methods of modeling the movement of people are used: steering behavior, models based on social forces, cellular automaton model, etc. Usually, as an input to evacuation models, the distribution of unimpeded travel speed is required, and then they use various methods, sub-models, algorithms for calculating speed with obstacles. When calculating the obstructive speed, two factors are mainly taken into account: 1) the interaction of the simulated evacuees with other evacuated people and 2) the interactions of those evacuated with the environment. Despite the fact that their use allows you to fix the relationship between the speed of movement, the density of people and the flow, there are additional factors that can affect the speed of movement with obstacles.

The article proposes a psychological model of the agent's behavior for the improvement of the multi-agent modeling method, which takes into account the individual characteristics of a person's personality, which is one of the factors affecting the speed of movement.

Keywords: multiagent modeling, agent model, evacuation modeling.

\author{
Киселев Сергей Сергеевич \\ Аспирант, ФГБОУ ВО «Юго-Западный \\ государственный университет» \\ kss89066905225@yandex.ru \\ Сазонов Сергей Юрьевич \\ К.т.н., дочент, ФГБОУ ВО «Юго-Западный \\ государственный университет» \\ serg_saz@mail.ru \\ Сазонова Екатерина Сергеевна \\ ФГБОУ ВО «Юго-Западный государственный \\ университет» \\ catasaz@mail.ru
}

Аннотация. Эвакуационные модели, используемые в технике пожарной безопасности, значительно расширили свои возможности в течение последних десятилетий. Они стали простыми вычислительными инструментами, в которых уравнения движения людей реализованы в дискретных пространствах, в то время как с помощью современных методов моделирования возможно моделировать сложное поведение и принятие решений людьми. Не смотря на прогресс методов эвакуационного моделирования при сравнении их возможностей кажется очевидным, что все ещё существует немало областей, в которых есть возможности для улучшений.

В эвакуационных моделях движение людей обычно основано на моделировании скорости беспрепятственной ходьбы, с которой передвигаются эвакуирующиеся, и влияющих на нее воздействий. Это один из ключевых факторов, оказывающих влияние на результаты, полученные спомощью моделей эвакуации. В настоящее время используются различные методы моделирования движения людей: поведение рулевого управления, модели на основе социальных сил, модель клеточного автомата и др. Обычно в качестве входных данных эвакуационным моделям требуется распределение беспрепятственной скорости передвижения, а затем они используют различные методы, подмодели, алгоритмы для расчета скорости с препятствиями. При расчете препятственной скорости в основном учитываются два фактора: 1) взаимодействие имитируемых эвакуированных с другими эвакуированными и 2) взаимодействия эвакуированных с окружающей средой. Не смотря на то, что их использование позволяет зафиксировать взаимосвязь между скоростью передвижения, плотностью людей и потоком, существуют дополнительные факторы, которые могут повлиять на скорость перемещения с препятствиями.

В данной статье предлагается психологическая модель поведения агента для усовершенствования мультиагентного метода моделирования, которая учитывает индивидуальные особенности личности человека, что является одним из факторов, влияющих на скорость передвижения.

Ключевые слова: мультиагентное моделирование, модель агента, эвакуационное моделирование. 


\section{Ввемение}

5 а последние десятилетия значительно увеличилось количество, возможности и использование в технике пожарной безопасности эвакуационных моделей[1]. Недавняя обзорная статья показала значительный рост числа публикаций в научных журналах, в которых принимаются во внимание эвакуационные модели[2]. С использованием моделей эвакуации были проведены различные типы исследований. Они сосредоточены на расширении возможностей модели и приложений модели в разных контекстах, эмпирических исследованиях валидации, вычислительной оптимизации и т.д. Рост научных исследований указывает на расширение сообщества эвакуационного моделирования. Также это может быть связано с принятием во всем мире кодов безопасности на основе характеристик[3], что, в свою очередь, связано с ростом числа применений эвакуационных моделей для техники пожарной безопасности.

Одной из основных проблем, связанных с эвакуационными моделями, является то, что они могут представлять определенные аспекты человеческого поведения, основанные на теоретических предположениях, а не на поведенческих данных[4]. Фактически, хотя эвакуационные модели в течение многих лет усовершенствовали свои возможности в представлении физических аспектов, связанных с пешеходными потоками, на сегодняшний день представление поведенческих аспектов эвакуации в значительной степени зависит от пользовательской компетенции модели, а не от применяемых методов моделирования. По этой причине в сообществе эвакуационного моделирования существует соглашение, согласно которому валидация модели должна быть одной из основных областей исследований эвакуации в будущем $[1,5]$. В настоящее время продолжаются обсуждения о процессе проверки эвакуационных моделей и необходимости увеличения собираемых наборов поведенческих данных при пожарной эвакуации[6, 7]. Недостаток таких данных связан с тем, что некоторые аспекты, касающиеся поведения человека, часто моделируются не управляемыми данными, а теоретическими допущениями (как, например, в случае доэвакуационного моделирования, которое сегодня редко явно реализуется в эвакуационных моделях, и в котором полагаются на распределение времени предварительной эвакуации). В настоящее время сообщество по эвакуационному моделированию решает данную проблему за счет различных усилий международных групп по сбору данных, которые участвуют в человеческом поведении при пожарных исследованиях.

Среди методов моделирования и методологий для проектирования и спецификации обычных программных систем существуют объектно-ориентированные подходы, основанные на центральном понятии объектов, которые инкапсулируют информацию состояния как совокупность значений данных и определяют поведение с помощью хорошо определенных интерфейсов для операций над этой информацией. Объектно-ориентированные методы определяют основные этапы идентификации, проектирования и переопределения объектов, позволяя абстрагироваться с помощью классов объектов и наследование в иерархии классов. Стремясь разработать методологию и модель, которая обеспечивает адекватную поддержку процесса разработки агентной системы, наш подход состоял в том, чтобы изучить, как существующие объектно-ориентированные методы моделирования могут быть расширены для применения к системам агентов. Объектно-ориентированные методы достигли значительной степени зрелости, и их преимущества получили широкое признание. Основываясь на существующих, хорошо понятных методах и адаптируя их, мы стремились разработать модель агента, которая будет легко узнаваема и понятна тем, кто знаком с парадигмой объектно-ориентированности.

Целью работы является усовершенствование метода мультиагентного моделирования с помощью разработки модели агента, которая будет учитывать психологические факторы, оказывающие влияние на поведение и мотивацию агента.

\section{1. Постановка заАачи}

Эвакуационные модели обычно предполагаются как самовозбуждающиеся системы частиц [8], то есть желаемая беспрепятственная скорость передвижения обычно представляет собой постоянное значение, полученное из распределения, которое затем уменьшается из-за разных факторов. В эмпирических и теоретических исследованиях вместо этого обсуждалось, что на желаемую скорость беспрепятственной ходьбы может влиять уровень мотивации каждого человека $[9,10]$. В данном случае мотивация представлена в контексте активирующего эффекта, т.е. скорость ходьбы имеет тенденцию к увеличению с повышением мотивации. Хотя это общая концепция, известная в других областях, например, в спортивной науке [11], этот вопрос не был тщательно исследован в эвакуационных исследованиях. В частности, мотивация может сыграть важную роль в определении индивидуальной или групповой желаемой скорости беспрепятственной ходьбы. Этот вопрос рассматривался в экспериментальных исследованиях, направленных на наблюдение скоростей ходьбы в нормальных и эвакуационных условиях [12]. В частности, мотивация может сыграть важную роль в определении индивидуальной или групповой желаемой беспрепятственной скорости ходьбы. Аналогичным образом, недавние экспериментальные исследования показали, что люди склонны уве- 
личивать скорость ходьбы в непосредственной близости от своего целевого назначения [13]. Представление человеческого поведения и сама движущая мотивация (т.е. фаза предварительного эвакуации) также связана с симуляцией восприятия риска, фундаментальным компонентом процесса пожарной эвакуации [14], который до сих пор редко принимался во внимание в эвакуации моделей. Например, были предприняты первоначальные попытки интегрировать восприятие риска и более сложные поведенческие переменные в модели принятия решений по эвакуации $[15,16]$, но для проведения таких калибровок для разных сценариев и условий необходимы дальнейшие экспериментальные исследования.

\section{2. Описание мохели агента}

При разработке агентной системы стоит принять во внимание более специализированный набор моделей, которые работают на двух разных уровнях абстракции. Во-первых, с внешней точки зрения система разбивается на агентов, смоделированных как сложные объекты, характеризуемые своим назначением, своими обязанностями, услугами, которые они выполняют, информацией, которая им необходима, и их внешними взаимодействиями. Во-вторых, с внутренней точки зрения, на элементы, которые требуются для конкретной архитектуры агента, и которые должны быть смоделированы для каждого агента. В нашем случае, это убеждения, цели и планы агента.

Описание системы агентов с внешней точки зрения фиксируется в двух моделях, которые в значительной степени не зависят от архитектуры.

- Модель агента описывает иерархическое отношение между различными абстрактными и конкретными классами агентов и идентифицирует экземпляры агента, которые могут существовать в системе, их множественность, а также момент, когда они появляются.

- Модель взаимодействия описывает обязанности класса агента, предоставляемые им услуги, связанные с ним взаимодействия и контролирует отношения между классами агентов. Сюда входят синтаксис и семантика сообщений, используемых для межагентной связи и связи между агентами и другими системными компонентами, такими как пользовательские интерфейсы.

С внутренней точки зрения каждый класс агента задается тремя моделями, соответствующими архитектуре, которые описывают его информационное и мотивационное состояние и его потенциальное поведение.

- Модель убеждений описывает информацию об окружающей среде и внутреннем состоянии, которые могут быть у агента этого класса, и дей- ствия, которые он может выполнять. Возможные убеждения агента и их свойства, например, могут ли они со временем меняться, описываются набором убеждений. Кроме того, может быть определено одно или несколько состояний убеждений - конкретные случаи набора убеждений и используемые для указания начального психического состояния агента.

- Модель цели описывает цели, которые может выбрать агент, и события, на которые он может ответить. Она состоит из набора целей, который определяет область цели и события, а так же одно или несколько состояний цели (совокупности целей цели), используемых для определения начального психического состояния агента.

- Модель плана описывает планы, которые агент может использовать для достижения своих целей или реагировать на события, которые он воспринимает. Он состоит из набора планов, который описывает свойства и структуру управления отдельными планами.

Одним из психологических факторов, оказывающих влияние на модель убеждений, является темперамент человека.

С психологической точки зрения существует 4 типа темперамента: флегматический, холерический, сангвинический и меланхолический. В экстренной ситуации люди с определенным темпераментом по-разному ведут себя.

Так как в природе не существует людей с четко выраженным определенным типом темперамента, то мы предлагаем наделять агента двумя типами темперамента.

Мы предполагаем введение коэффициента наступления паники (р) в зависимости от темпераментов. В случае, если при проверке этот коэффициент больше определенного значения, то агент впадает в паническое состояние. Так как паника проявляется в различных формах (от полного бездействия до неупорядоченных действий), то мы предполагаем введение ещё одного коэффициента (n), в зависимости от значения которого будет выбираться тип панического состояния. Проверка коэффициентов будет проводиться при каждой итерации моделирования системы.

\section{3. Алгоритм молелирования агента}

На рисунке 1 представлена блок-схема алгоритма поведения моделируемого агента.

На этапе создания агент наделяется физическими характеристиками (возраст, мобильность, занимаемая 


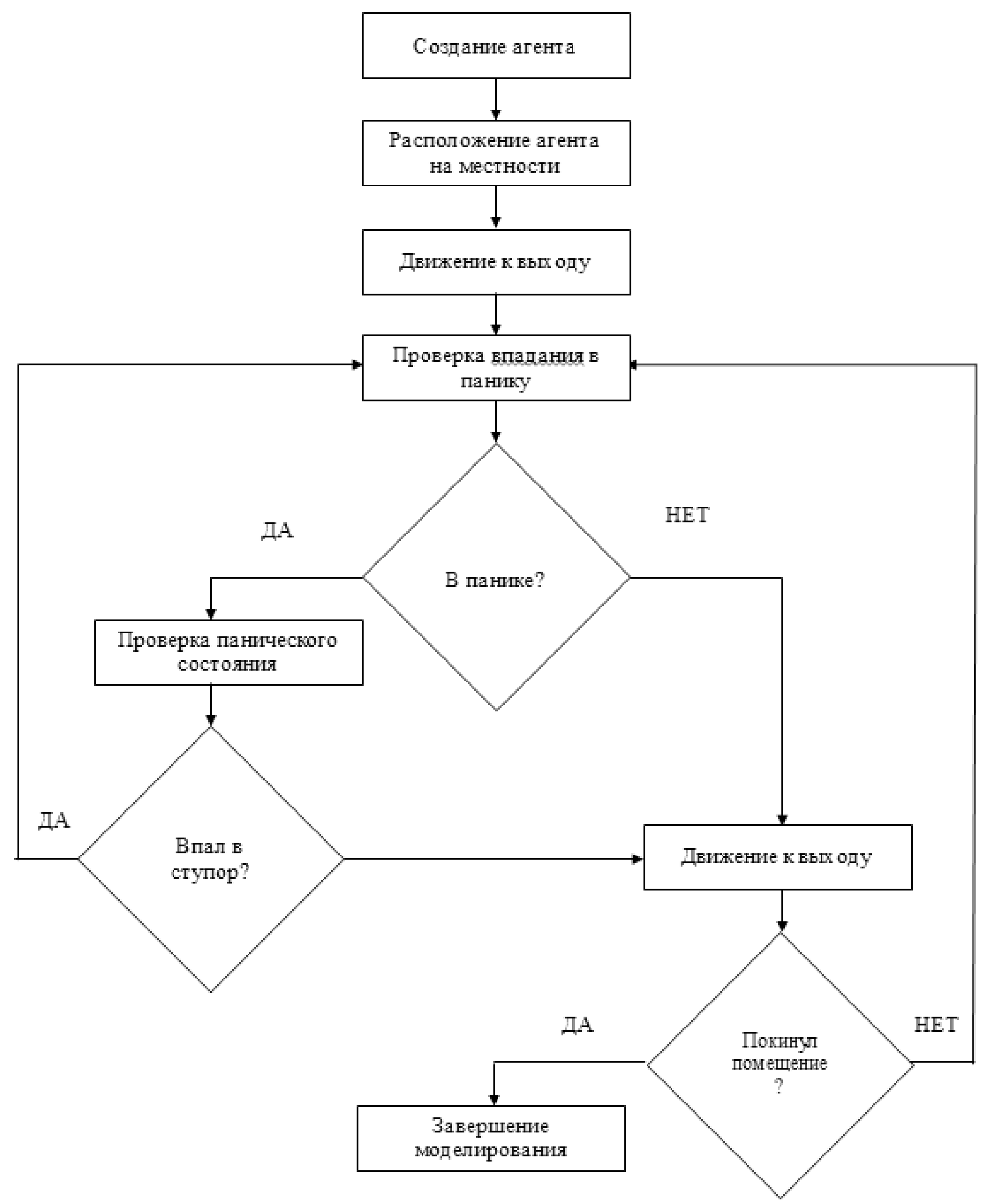

Рис. 1. Блок-схема алгоритма поведения моделируемого агента 
площадь, скорость передвижения), убеждениями, целью (эвакуироваться из помещения), планом (выбор маршрута эвакуации), темпераментом (случайно выбираются 2 типа темперамента и случайно устанавливается их процентное соотношение).

На этапе расположения агента на местности агент случайно располагается в созданном помещении.

Далее агент начинает двигаться в сторону выхода в соответствии с правилами своего плана.

На основе соотношения темпераментов (коэффициент р) каждую секунду моделирования проводится проверка состояния агента.

В том случае, если агент впал в панику, то проводится проверка панического состояния агента (коэффициент п) и изменяется его скорость передвижения. Если агент впал в ступор, то скорость передвижения становится равной 0 и агент ожидает следующей проверки состояния, так как существует вероятность, что агент успокоится и начнет движение к выходу. Если же агент не впал в ступор, то его скорость изменяется случайным образом и он продолжает движение к выходу.

Если при проверке агент не впал в панику, то он продолжает движение к выходу с неизменной скоростью.
В том случае, если агент покинул помещение, то он удаляется из модели и процесс моделирования агента завершается.

\section{Зак^ючение}

Предлагаемая модель может быть использована для модификации систем управление эвакуацией людей, основанных на мультиагентных методах моделирования. Так как модель принимает во внимание убеждения, цели и планы агента, то при моделировании появляется возможность описать потенциального человека наиболее полно с психической точки зрения.

Учет при моделировании психической особенности личности - темперамента, и его влияние на возможность человека впасть в паническое состояние, позволяет оценивать полное время эвакуации с большой приблеженностью к реальности, поскольку в любой момент времени человек может впасть в ступор или изменить направление движения относительно выхода в другую сторону.

Однако данная модель принимает во внимание только один личностный психический фактор. Для улучшения модели следует внедрить другие личностные характеристики и тем самым усовершенствовать моделирование поведения людей при эвакуации.

\section{ЛИТЕРАТУРА}

1. Enrico Ronchi, and Michael Kinsey. "Evacuation Models of the Future: Insights from an Online Survey on User's Experiences and Needs." In, 145-155. Capote, J. et al, Santander, Spain. 2011.

2. Hendrik Vermuyten, Jeroen Beliën, Liesje De Boeck, Genserik Reniers, and Tony Wauters. "A Review of Optimisation Models for Pedestrian Evacuation and Design Problems." Safety Science 87 (August): 167-178. Aug. 2016.

3. Brian J. Meacham. "Fire Safety Engineering at a Crossroad." Case Studies in Fire Safety 1 (March): 8-12. Mar. 2014.

4. S.M.V. Gwynne, L. M. Hulse, and M. J. Kinsey. "Guidance for the Model Developer on Representing Human Behavior in Egress Models." Fire Technology, May. May 2015.

5. Enrico Ronchi." "The Need for a Verification and Validation Protocol for Evacuation Models." In. Gaithersburg, MD (US). 2014.

6. J.D. Averill." "Five Grand Challenges in Pedestrian and Evacuation Dynamics." In Pedestrian and Evacuation Dynamics, edited by Richard D. Peacock, Erica D. Kuligowski, and Jason D. Averill, 1-11. Springer US, Boston, MA. 2011.

7. Enrico Ronchi, Erica D. Kuligowski, Paul A. Reneke, Richard D. Peacock, and Daniel Nilsson. "The Process of Verification and Validation of Building Fire Evacuation Models. Technical Note 1822." National Institute of Standards and Technology. 2013.

8. Tamás Vicsek, András Czirók, Eshel Ben-Jacob, Inon Cohen, and Ofer Shochet. "Novel Type of Phase Transition in a System of Self-Driven Particles." Physical Review Letters 75 (6): 1226-1229. Aug. 1995

9. Ethel Graat, Cees Midden, and Paul Bockholts. "Complex Evacuation; Effects of Motivation Level and Slope of Stairs on Emergency Egress Time in a Sports Stadium." Safety Science 31 (2): 127-141. Mar. 1999. doi:10.1016/S0925-7535(98)00061-7.

10. Enrico Ronchi, Paul A. Reneke, and Richard D. Peacock. "A Conceptual Fatigue-Motivation Model to Represent Pedestrian Movement during Stair Evacuation."Applied Mathematical Modelling 40 (7-8): 4380-4396. Apr. 2016.

11. Robert J Vallerand, and Gaétan F Losier. “An Integrative Analysis of Intrinsic and Extrinsic Motivation in Sport." Journal of Applied Sport Psychology 11 (1): $142-169$. 1999.

12. CJ Khisty. Pedestrian Flow Characteristics on Stairways during Disaster Evacuation. HS-040 034. 1985.

13. Enrico Ronchi, Johan Norén, Mattias Delin, Kalev Kuklane, Amitava Halder, Silvia Arias, and Karl Fridolf. Ascending Evacuation in Long Stairways: Physical Exertion, Walking Speed and Behaviour. 3192. Department of Fire Safety Engineering, Lund University, Lund, Sweden. 2015. 
14. Max T. Kinateder, Erica D. Kuligowski, Paul K. Reneke, and Richard D. Peacock. A Review of Risk Perception in Building Fire Evacuation. NIST TN1840. National Institute of Standards and Technology. Sep. 2014.

15. Ruggiero Lovreglio, Enrico Ronchi, and Daniel Nilsson. "A Model of the DecisionMaking Process during Pre-Evacuation." Fire Safety Journal 78 (November): $168-179$. Nov. 2015. doi:10.1016/j.firesaf.2015.07.001.

16. Ruggiero Lovreglio, Enrico Ronchi, and Daniel Nilsson. "An Evacuation Decision Model Based on Perceived Risk, Social Influence and Behavioural Uncertainty." Simulation Modelling Practice and Theory 66 (August): 226-242. Aug. 2016.

( ) Киселев Сергей Сергеевич ( kss89066905225@yandex.ru ),

Сазонов Сергей Юрьевич ( serg_saz@mail.ru ), Сазонова Екатерина Сергеевна ( catasaz@mail.ru ).

Журнал «Современная наука: актуальные проблемы теории и практики»

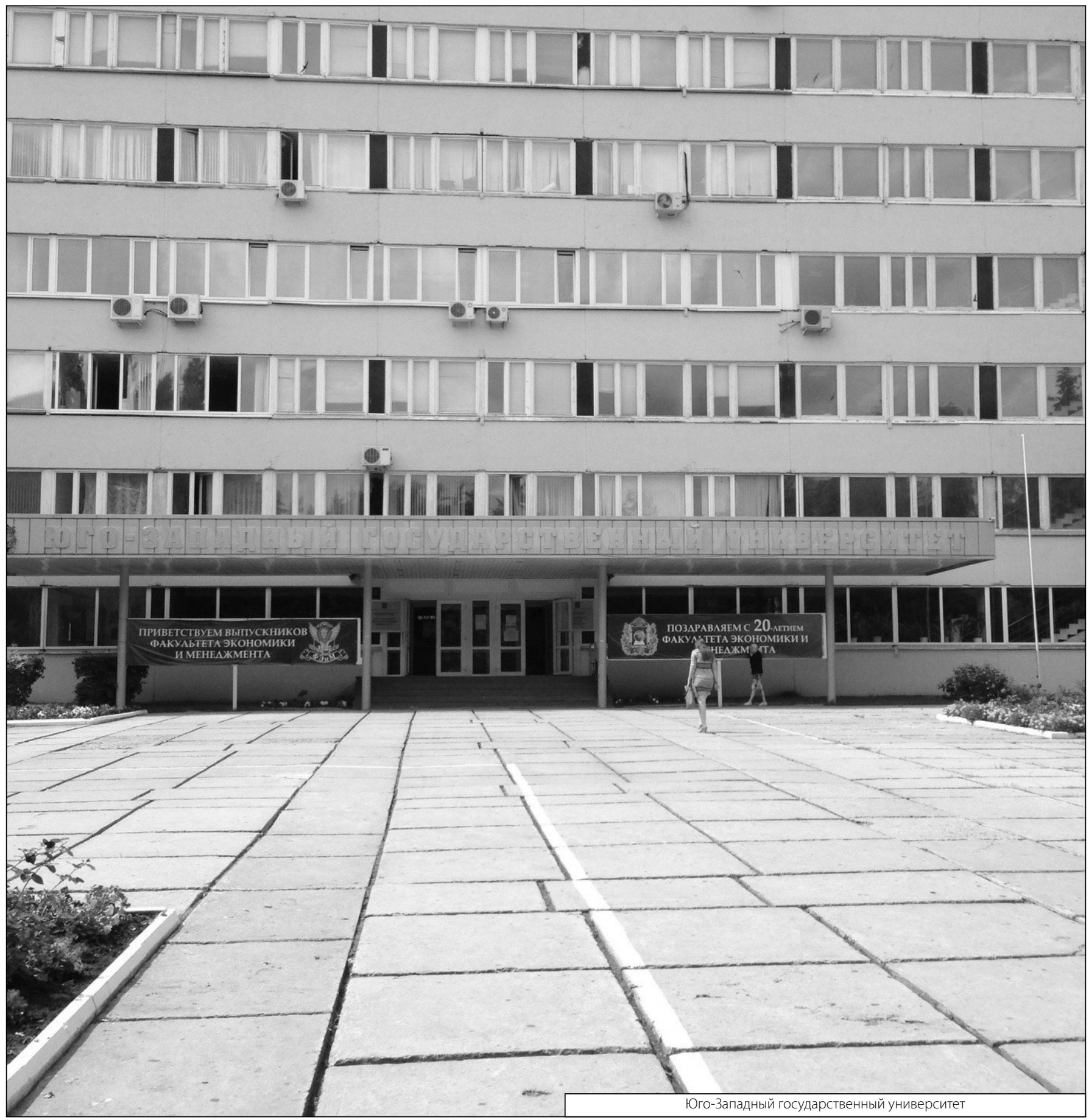

